\title{
Location-based Robust Beamforming Design for Cellular-enabled UAV Communications
}

\author{
Wang Miao, Chunbo Luo, Geyong Min, Yang Mi, and Zhengxin Yu \\ College of Engineering, Mathematics and Physical Science, University of Exeter, UK
}

\begin{abstract}
Cellular communications have been regarded as promising approaches to deliver high-broadband communication links for Unmanned Aerial Vehicles (UAVs), which have been widely deployed to conduct various missions, e.g. precision agriculture, forest monitoring and border patrol. However, the unique features of aerial UAVs including high-altitude manipulation, three-dimension (3D) mobility, and rapid velocity changes, pose challenging issues to realize reliable cellular-enabled UAV communications, especially with the severe inter-cell interference generated by UAVs. To deal with this issue, we propose a novel position-based robust beamforming algorithm through complementarily integrating the navigation information and wireless channel information to improve the performance of cellularenabled UAV communications. Specifically, in order to achieve the optimal beam weight vector, the navigation information of the UAV system is innovatively exploited to predict the changes of Direction-of-arrival (DoA) angle. To fight against the high mobility of UAV operations, an optimization problem is formed by considering the tapered surface of DoA angle and solved to correct the inherent position error. Comprehensive simulation experiments are conducted and the results show that the proposed robust beamforming algorithm could achieve over 90\% DoA estimation error reduction and up to 14dB SINR gain compared with five benchmark beamforming algorithms, including Linearly Constrained Minimum Variance (LCMV), Position-based beamforming, Diagonal Loading (DL), Robust Capon Beamforming (RCB) and Robust LCMV algorithm.
\end{abstract}

Index Terms-Cellular Communications, Unmanned Aerial Vehicle, Robust Beamforming, Direction-of-Arrival, GPS Navigation

\section{INTRODUCTION}

Recent years have witnessed the rapid development of Unmanned Aerial Vehicles (UAVs) and their explosive usages in human society. For instance, UAVs have been used to deliver medicine and vaccines into and out of remote or inaccessible regions [1]. UAV also have been used to patrol the border and monitor the oil pipeline [2]. Wireless communication is critically important for UAVs to accomplish their missions. The Control and Command and airborne camera/sensor data need to be securely and reliably sent through wireless communication to enable safe flight and swift task accomplishment. Owning to their merits of wide deployment, high speed and stable transmission links, cellular communications such as the existing cellular and the coming Fifth Generation (5G) networks, have been regarded as the most potential candidates to offer high broadband wireless services and out-of-sight control and monitoring for UAV flight and mission execution.

For cellular-enabled UAV communications, there are two challenges that need to be carefully considered and addressed. Firstly, the patterns that Ground User Equipment (GUE) and Aerial User Equipment (AUE) are served by cellular networks are quite different. For terrestrial wireless communication, GUEs consume more downlink bandwidth for watching videos, browsing online contents and viewing social networks. While in the sky, AUEs become the sources of high-definition videos and require high-performance wireless networking system to transmit these visual data. The design of cellular network architecture that caters to downlink transmission does not pay enough attention to the potential requirements from the uplink transmission. Secondly, due to the lack of obstacles and widely existing Line of Sight (LoS) paths, AUEs produce more uplink interference (UAV to Base Station (BS)) than ground mobile devices, significantly affecting the performance of wireless communications. In addition, UAVs are equipped with high-resolution cameras to capture High Definition (HD) video, which facilitates the operations of spotting a missing person and identifying the slight corrosion of building or bridges, the crevice of oil pipelines or any damages of telecommunication facilities. However, the value of all those data cannot be fully explored if they are not delivered to the underground control centre in time.

Receiving beamforming has been considered a promising technology for cellular communication to address the serves interference issue and support the emerging UAV applications [3] [4] [5] [6] [7]. Through adaptively adjusting the antenna radiation patterns, the array elements at BS could be combined constructively or destructively to form the peaks and nulls in the antenna beam, which provides the benefits of simultaneously enhancing the signal transmission and mitigating the adverse interferences [8]. The accurate Direction-of-arrival (DoA) estimation plays an important role in beamforming algorithms to achieve the optimal antenna response. While traditional beamforming algorithms suffer from serious performance degradation due to the inaccurate DoA estimation caused by the high mobility of UAV operations. To address this issue, tremendous research efforts have been made to design high-performance robust beamforming algorithms, e.g. Diagonal Loading [9], Robust Capon Beamforming [10], and robust Linearly Constrained Minimum Variance (LCMV) [11]. Through the introduction of noise factors in the variance function of the input signal, robust beamforming algorithms are able to improve the DoA error tolerance through broadening the main-lobe of antennas, however, at the cost of degraded output power and Signal to Interference plus Noise Ratio (SINR), and struggle to satisfy the high-broadband requirements of UAV applications. Therefore, how to enhance the design of robust beamforming becomes urgent and important 
for cellular networks to realise stable UAV communications.

To address these issues, we propose a novel robust beamforming algorithm to efficiently cancel the inter-cell interference and boost the system throughput for the cellular-enabled UAV communication system. The major contributions of this work are summarised as follows:

- Through complementarily integrating the navigation information of UAV system and DoA information of wireless communication system, a new robust beamforming optimization problem is formed and solved with the aim of maximizing the output SINR under the constraint of strong interference.

- To make the optimal beam weight vector tolerate to DoA errors, we exploit the UAV position and mobility information to track UAV, predict the changes of DoA angle, adaptively adjust the beamforming weights and realize optimal signal reception. A mathematical analysis is conducted to theoretically investigate the effects of DoA error on the output power of the beamforming algorithm.

- To fight against the dynamic mobility of UAV operation, an optimization problem is established and solved to correct position errors through minimizing the distance between error positions and the tapered surface formed by DoA angle. By cooperatively utilizing navigation and DoA information, the proposed robust beamforming algorithm could achieve higher output power and SINR.

- To evaluate the performance of the proposed algorithm, extensive simulation experiments are conducted and the results show that the proposed algorithm achieves over $90 \%$ DoA error reduction and up to $14 d B$ SINR gains compared with the well-known robust LCMV algorithm and position-based beamforming without position correction.

The rest of this paper is organized as follows. Section II presents the state-of-the-art of receiving beamforming. Section III discusses the network architecture and system model. Section IV proposes a novel position-based robust beamforming algorithm to track UAV positions and enhance the throughput for cellular-enabled UAV communication. The performance of the proposed algorithm is validated in Section V. Finally, Section VI concludes this paper.

\section{STATE-OF-THE-ART}

Receiving beamforming is a promising technology for cellular communication to increase the SINR of signal reception, reduce inter-cell interference and enhance overall system performance. Due to its superior performance improvement, receiving beamforming has been considered as one of the most important key technologies in $5 \mathrm{G}$ communication together with MIMO, small cell, millimetre wave communications software defined networking and network function virtualisation to realize its 1000 times throughput improvement [12] [13] [14] [15]. Therefore, tremendous research efforts have been made in the area of beamforming design. For example, the authors in [5] designed a MIMO conjugate beamforming algorithm to effectively improve the performance of the terrestrial cellular network and enhance the spectrum efficiency. Ramy et al. [16] proposed a novel system framework of cooperation transmission to support reliable connectivity and developed a new mobility model to capture the omnipresent mobility of UAVs. The experiment results show that the proposed solutions could significantly improve the coverage probabilities of UAV system, from $28 \%$ to $60 \%$, nearly 2.5 times performance enhancement. In addition, the authors in [7] proposed a novel cell-free massive MIMO architecture to support the communications of UAVs in the air. With the assumption of the Rican channel, this work discusses the strategies to allocate transmission power and radio resources, jointly considering the allocation fairness and spectrum efficiency. The minimum output energy (MOE) beamformer in [17] defines an optimization criterion to minimize the total output energy while simultaneously keeping the gain of the array on the desired signal fixed. Because the gain on the signal is fixed, any reduction in the output energy is obtained by suppressing the interference and noise. Minimum variance distortionless response (MVDR) is a special form of MOE beamformer and obtained by setting the gain of the desired signal as a constant value, which means the desired signal is not distorted during minimizing the output signal power. Different from MOE and MVDR focusing on output power, the authors in [18] designed a successive multiuser beamforming scheme in a multi-cell cooperative uplink transmission to maximize the SINR to meet the transmission requirement for each user. These beamforming algorithms could achieve optimal antenna responses, and therefore are called as the optimal beamformers in wireless communication.

Although optimal beamformers could potentially achieve the optimal performance by isolating the interesting signal from interference and noise, to explore them for UAV data communications meets significant challenges. Because optimal beamforming algorithms require the advanced acquisition and tracking technologies to ensure that signal sources are covered by the main beams and have high SINR for information transmission. For example, optimal beamformers assume that the DoA could be accurately estimated and achieved. However, due to the unique features of UAV operations, e.g. high mobility and 3D movement, it is quite difficult to accurately estimate the DoA, which makes the existing optimal beamforming algorithms suffer from serious performance degradation. To address this issue, blind robust beamforming algorithms, e.g. Diagonal Loading [9], Robust Capon Beamforming [10], and robust LCMV [11], have been proposed. Through the introduction of noise factors in the variance function of the input signal, robust beamforming algorithms are able to broaden the antennae's main-lobe and improve the DoA error tolerance, however, at the cost of degraded output power and SINR. For example, recent research work [19] revealed that the navigation and mobility information of UAVs universally exists at the ground control centre, BSs even remote cloud for UAV safety management. Comparing with the robust beamforming strategies, UAV's navigation and mobility information can be utilized to compensate for DoA errors, adaptively adjust the beamforming weights and realize optimal signal reception. The functions of GPS and mobility information in the beamforming algorithm are to calculate and predict the DoA angles when 
UAV is operated in the sky.

Actually, the idea of exploiting the position information to conduct beamforming has been used in the ground mobile communication systems to support vehicles and trains. For example, Xiong et al. [16] exploited the vehicles current position and velocity information to design a broad beamforming approach to improve the Signal-to-Noise Rate (SNR). In addition, the authors in [20] developed a location informationbased beamforming algorithm to leverage cellular networks to support the communication services of high-speed railway. The proposed algorithm pre-calculates the beamforming weights based on the train location information and can be completed through an off-line calculation, significantly reducing the complexity of the algorithm design and implementation. Although some interesting results have been produced in the area of the position-based beamforming design, the existing studies can be hardly used to support the cellularbased UAV communications. Because they didn't consider the unique features of UAV applications e.g. 3D mobility, interference cancellation, position error and so on. According to the test results [21], the number of cells receiving the interference is increasing with the growth of the altitudes that UAVs are operating. The range and strength of the interference in cellular-enabled UAV communication are much larger than that of the traditional cellular communication. The interference cancellation should not only consider the interference sources from its neighboring cell, but also the remote cells. In addition, different from the 2D fixed tracks of the trains or vehicles, e.g. rails, roads or bridges, UAVs are operated in the sky with dynamic speeds and directions, calling for efficient methods to handle the position error caused by operational dynamics.

To bridge this gap, this paper proposes a position-based robust beamforming algorithm for cellular-based UAV communications. To the best of our knowledge, interference cancellation coupled with the position error correction has not been reported in the existing literature. In detail, a new beamforming algorithm is designed with the aim of cancelling the interference from the UAVs in the neighbor and maximizing the overall system throughput. Furthermore, to fight against the dynamic mobility of UAV flight, a position correction method is developed in this work to minimize the position error. The mechanisms of the beamforming algorithm design and the position correction method will be introduced in Sections III and IV.

\section{Network ARchitecture AND Model DESCRIPTION}

In this section, we first describe a general network architecture for cellular network to support UAV system and then present the system model of beamforming design in cellularenabled UAV communications.

\section{A. Network Architecture}

Fig. 1 shows a holistic network architecture of cellularenabled UAV communication system, which consists of $B_{c}$ eNode BS (eNBs) stations with distinct cell IDs $c^{b}$, where $c^{b} \in N_{B_{c}} . N_{B_{c}}$ denotes the subset of natural numbers with

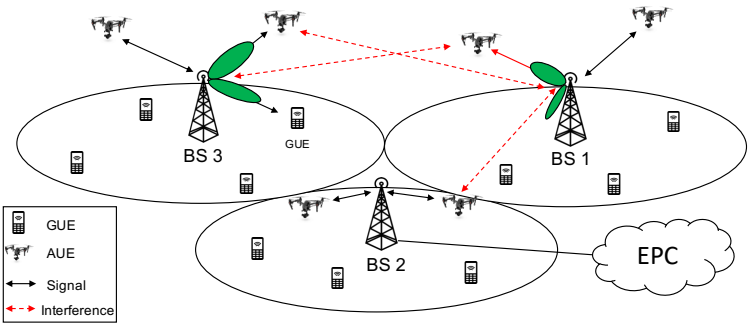

Fig. 1: Network architecture of cellular-enabled UAV communications (GUE: Ground User Equipment; AUE: Aerial User Equipment; BS: Base Station; and EPC: Evolved Packet Core)

cardinality $B_{c}$. There are $N_{c^{b}}^{a}$ AUEs served by the $c^{b}$ th eNB together with $N_{c^{b}}^{g}$ GUEs. Compared with the traditional cellular system, the $c^{b}$ th eNB needs to guarantee normal wireless mobile services for ground GUEs, at the same time offers highperformance wireless services for AUEs. The first challenging issue for realizing UAV-supported cellular networks is the serious interference generated by AUEs. According to the research results published by Ericsson [22], AUEs produce more uplink interference in the cellular network than GUEs because free space propagation increases the probability that signals could be transmitted and received by neighbour cells. Since both the AUEs and GUEs will coexist and be served by cellular networks, the first requirement for the beamforming algorithm to be designed is the high capability of strong interference cancellation. In addition, bandwidth-intensive applications will be transmitted from the AUE to eNB, the beamforming algorithm should be developed to maximize the output throughput and provide high broadband to meet transmission requirements.

The communications between AUEs and BS involve two kinds of wireless channels, Ground to UAV (G2A) and UAV to Ground (A2G) [23], exhibiting different transmission characteristics. For instance, G2A is mainly used to deliver the flight command or task message, requiring stable and reliable transmissions. While the $\mathrm{A} 2 \mathrm{G}$ is in charge of high-volume of data delivery, consuming huge channel bandwidth and has various delay requirements for different applications. In this work, the robust beamforming will be designed to improve the performance of the uplink transmission. However, due to its feature of utilizing position information to estimate DoA, the proposed algorithm can also be applied to the downlink wireless communication transmission.

\section{B. System Model}

Let $x_{0}(k)$ and $x_{i}(k)$ present the interesting and interfering signal received by the $c^{b}$ th eNB at the $k$ th resource allocation slot. Then the receiving signal of antenna system $s(k)$ could be expressed as [24]

$$
s(k)=\alpha\left(\theta_{0}\right) x_{0}(k)+\sum_{i=1}^{N_{i}} \alpha\left(\theta_{i}\right) x_{i}(k)+n(k),
$$

where $N_{i}$ denotes the number of the interfering sources and $\alpha\left(\theta_{0}\right)$ and $\alpha\left(\theta_{i}\right)$ are the antenna steering vector for the interesting and the $i$ th interfering signals with DoAs of $\theta_{0}$ and $\theta_{i} . n(k)$ stands for the additive white Gaussian noise at 
the BS. For $i \in N_{i}, \alpha\left(\theta_{0}\right)$ and $\alpha\left(\theta_{i}\right)$ are calculated by the following equation,

$\alpha(\theta)=\left[1, \exp \left\{-j \frac{2 \pi}{\lambda} d \sin \theta\right\}, \ldots, \exp \left\{-j \frac{2 \pi}{\lambda} d(L-1) \sin \theta\right\}\right]^{T}$,

where $L, \lambda$ and $d$ denote the antenna number, signal wavelength and distance between two antenna elements. Let $A(\theta)=\left[\alpha\left(\theta_{0}\right), \alpha\left(\theta_{1}\right), \ldots, \alpha\left(\theta_{N}\right)\right]$ and $x(t)=$ $\left[x_{0}(k), x_{1}(k), \ldots, x_{N}(k)\right]$, then Eq. (1) could be transformed to $s(t)=A(\theta) x(t)^{T}+n(k)$. Given a beamforming weight vector, $\omega$, the output signal, $y(t)$, is calculated as

$$
y(k)=\omega^{H} s(k) \text {. }
$$

\section{Robust BeAmforming DESIGN}

\section{A. Beamforming Design}

By exploiting the navigation information from GPS and IMU, this subsection aims at designing a new robust beamforming algorithm to satisfy the performance requirements of the data transmission. The proposed algorithm is to maximize the output throughput, realize the interference cancellation and fight against the high mobility of the AUEs. The output signal is shown as

$$
y(k)=\omega^{H}\left[\alpha\left(\theta_{0}\right) x_{0}(k)+\sum_{i=1}^{N} \alpha\left(\theta_{i}\right) x_{i}(k)+n(k)\right] .
$$

And the SINR of $y(k), \rho$, can be calculated by [24]

$$
\rho=\frac{E\left\{\left|\omega^{H} \alpha\left(\theta_{0}\right) x_{0}(k)\right|^{2}\right\}}{\sum_{i=1}^{N} E\left\{\left|\omega^{H} \alpha\left(\theta_{i}\right) x_{i}(k)\right|^{2}\right\}+E\left\{\left|\omega^{H} n(k)\right|^{2}\right\}} .
$$

where $\alpha\left(\theta_{0}\right)$ and $\alpha\left(\theta_{i}\right)$ are the antenna steering vector for the interesting and the $i$ th interfering signals with DoAs of $\theta_{0}$ and $\theta_{i}$.

According to the Shannon Theorem [25], the throughput, $\mathbb{R}$, is a function of output SINR shown as

$$
\mathbb{R}=B \log _{2}(1+\rho) .
$$

Therefore, the maximization of the throughput could be guaranteed by maximizing the output SINR under the resources restriction (bandwidth $B$ ), described as $\max (R)=\max (\rho)$. Denote the power of the signals from the interested and interference UAVs as $P_{0}$ and $P_{i}$, then the maximization of the output SINR is described as

$$
\begin{aligned}
& \max \frac{E\left\{\left|\omega^{H} \alpha\left(\theta_{0}\right) x_{0}(k)\right|^{2}\right\}}{\sum_{i=1}^{N} E\left\{\left|\omega^{H} \alpha\left(\theta_{i}\right) x_{i}(k)\right|^{2}\right\}+E\left\{\left|\omega^{H} n(k)\right|^{2}\right\}} \\
& =\max \frac{P_{0} E\left\{\left|\omega^{H} \alpha\left(\theta_{0}\right)\right|^{2}\right\}}{\sum_{i=1}^{N} P_{i} E\left\{\left|\omega^{H} \alpha\left(\theta_{i}\right)\right|^{2}\right\}+E\left\{\left|\omega^{H} n(t)\right|^{2}\right\}} .
\end{aligned}
$$

It should be noticed that Eq. (7) aims to maximize the SINR of beamforming output at the BS. Towards this aim, we transform Eq. (7) to the following optimization problem,

$$
\begin{gathered}
\omega_{o p t}=\arg \min E\left[\left|\omega^{H} R_{n} \omega\right|^{2}\right], \\
\text { s.t. }\left\{\begin{array}{l}
\omega^{H} \alpha\left(\theta_{0}\right)=1, \\
\omega^{H} \alpha\left(\theta_{i}\right)=0, \quad i=1,2, \ldots, N .
\end{array}\right.
\end{gathered}
$$

where $R_{n}$ is the autocorrelation matrix of the noise received by the BS. The aim of Eq. (8) is to find an optimal beamforming vector, which maximizes the antenna response in the direction of the signal source (the denominator of Eq. (7)) and minimizes the response for the interference sources (the numerator of Eq. (7)). By utilizing the Lagrange Multiplier [26] (see APPENDIX A), the optimal beamforming weight could be calculated by

$$
\omega=-\left[A(\theta)^{H} R_{n}^{-1} A(\theta)\right]^{-1} R^{-1} A(\theta) F,
$$

where $F=[1,0, \ldots, 0]$.

\section{B. DoA Error Analysis}

To achieve the optimal performance of beamforming design, one of the key issues is to calculate the accurate DoA information from the signal received. However, due to the high mobility of the UAV system, the accuracy of the DoA estimation is greatly affected by the UAV flight status and channel condition. In this subsection, we analyze DoA errors and their impacts on the output power of the optimal beamforming algorithm. In the next subsection, a position-based DoA correction method is proposed to reduce DoA error, which is very important for achieving optimal performance for beamforming design. Let $\alpha_{t}(\theta)+\Delta \alpha(\theta)$ denote the steering vector with an error $\Delta \alpha(\theta)$. Given the power of the arrival signal as $P$, then the output power of the beamforming algorithm is given by

$$
P_{s}=P \omega^{H} A(\theta)^{H} A(\theta) \omega .
$$

Eq. (10) calculates the output power under the accurate DoA estimation and Eq. (9) provides the method to calculate the optimal beamforming weight. While, due to the long distance transmission and GPS signal fading, there is inherent position error in GPS coordinate information, which could result in the inaccurate DoA estimation and affect the performance of the optimal beamforming algorithm in Eq. (9). In this case, the beamforming weight, $\omega$ could be updated as

$$
\begin{array}{r}
\omega_{e}=\left\{[A(\theta)+\Delta \alpha(\theta)]^{H} R_{n}{ }^{-1}[A(\theta)+\Delta \alpha(\theta)]\right\}^{-1} * \\
R_{n}{ }^{-1}[A(\theta)+\Delta \alpha(\theta)] F .
\end{array}
$$

Under the estimated steering vector, the output power of the beamforming algorithm is calculated by

$$
P_{e}=P \omega_{e}^{H} A(\theta)^{H} \alpha_{t}(\theta) \omega_{e} .
$$

Due to the existence of DoA error, the output power loss, $P_{l}$, can be defined as

$$
P_{l}=P_{s}-P_{e}
$$

By taking the values of $\omega_{e}, \omega, \alpha_{t}$ and $\Delta \alpha(\theta)$ into Eq. (13), the output power loss can be calculated as

$$
\begin{aligned}
P_{l}= & P_{s}\left(\mu^{2}-\mu_{1}^{2}\right) A(\theta)^{H}\left(R_{n}^{-1}\right)^{H} \Delta \alpha(\theta) A(\theta)^{H} R_{n}{ }^{-1} \Delta \alpha(\theta)- \\
& P_{s} \mu_{1}^{2}\left(R_{n}^{-1}\right)^{H} \Delta \alpha(\theta) A(\theta)^{H} R_{n}^{-1} * \\
& {\left[A(\theta) A(\theta)^{H}+A(\theta)(\Delta \alpha(\theta))^{H}+(\Delta \alpha(\theta))^{H} \Delta \alpha(\theta)\right] . }
\end{aligned}
$$


where $\mu$ and $\mu_{1}$ are calculated by $\mu=\left[A(\theta)^{H} R_{n}{ }^{-1} A(\theta)\right]^{-1}$ and $\mu_{1}=\left[(A(\theta)+\Delta \alpha(\theta))^{H} R_{n}^{-1}(A(\theta)+\Delta \alpha(\theta))\right]^{-1}$.

The DoA error $\Delta \theta$ is closely related to the UAV flight status. The same moving direction, speed and acceleration would result in different DoA errors when the UAVs are operated at different horizontal and vertical distances from the BS. For instance, when the UAV is near to the BS, the communication system would experience large scale of DoA errors. While, when the UAV is far from the BS, small DoA error would be introduced to the steering vector. The relationship between the DoA estimation at the $k$ th resource allocation slot and the position and movement information is given by the following equation,

$$
\theta_{k}=\arctan \left(\frac{\sqrt{x_{k}^{2}+\left(z_{k}-z_{k}^{b}\right)^{2}}}{y_{k}}\right),
$$

where $\left(x_{k}, y_{k}, z_{k}\right)$ is the three-dimensional position information at the $k$ th moment and $z_{k}^{b}$ is the height of the BS. Let $\left(v_{k-1}^{x}, v_{k-1}^{y}, v_{k-1}^{z}\right),\left(a_{k-1}^{x}, a_{k-1}^{y}, a_{k-1}^{z}\right)$, and $\tau$ denote the speed and acceleration of the $(k-1)$ th transmission and time interval between the $k$ th and $(k-1)$ th transmissions. Then the position update could be calculated in the following equation,

$$
\left[\begin{array}{l}
x_{k} \\
y_{k} \\
z_{k}
\end{array}\right]^{T}=\left[\begin{array}{l}
x_{k-1} \\
y_{k-1} \\
z_{k-1}
\end{array}\right]^{T}+\tau\left[\begin{array}{l}
v_{k-1}^{x} \\
v_{k-1}^{y} \\
v_{k-1}^{z}
\end{array}\right]^{T}+\tau^{2} *\left[\begin{array}{l}
a_{k-1}^{x} \\
a_{k-1}^{y} \\
a_{k-1}^{z}
\end{array}\right]^{T} .
$$

Eqs. (15) and (16) provide the methods to calculate the DoA from the coordinate and how to update the coordinate between two resource allocation slots. As there are inevitable errors in the GPS coordinate and same amount of GPS coordinate error would result in different DoA estimation error when UAV are at different spatial positions. In order to reveal the relationship between the DoA estimation errors and the spatial positions, we conducted simulation experiments by setting the GPS error as $10 \mathrm{~m}$. UAV is operating at the horizontal distances of $10 \mathrm{~m}$, $50 \mathrm{~m}, 100 \mathrm{~m}, 150 \mathrm{~m}$, respectively, and the vertical altitudes in the range of $[0,300 \mathrm{~m}]$. The height of BS is set to be $15 \mathrm{~m}$. The results are collected every $10 \mathrm{~m}$ in the vertical direction and are drawn in Fig. 2. It should be noticed that we adopt a $10 \mathrm{~m}$ fixed GPS error in the simulation configuration and a determinate DoA estimation error is obtained from the simulation. Instead of adopting random GPS errors, such fixed GPS error configuration helps us to obtain a smooth curve to clearly show the trend of the DoA estimation errors with the increase of the UAV altitudes at different horizontal distances. From Fig. 2, it can be seen that the position-based DoA estimation approach suffers from serious estimation error when UAV is flying near to BS and has little DoA error when UAV is flying far from the BS. It should be noticed that we adopt a 10m fixed GPS error in the simulation configuration, which would result in shown in determinate DoA estimation errors as shown in Fig. 2. This setting could help us to obtain a smooth curve to clearly show the trend of the DoA estimation error with the increase of the UAV operating altitudes at different horizontal distances. In addition, there is a horizontal line at each curve in Fig. 2. This is because 10m GPS position error

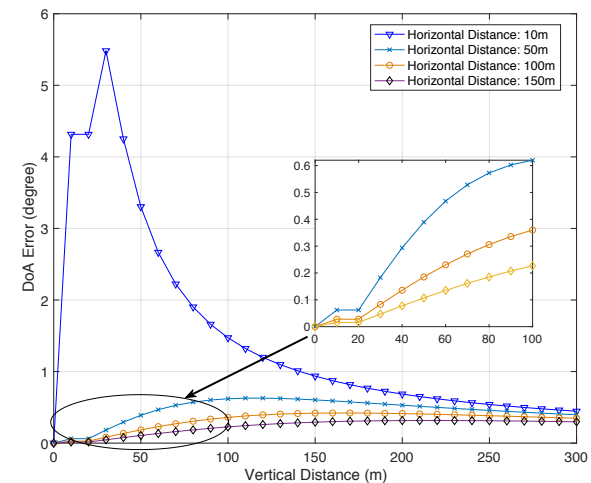

Fig. 2: DoA error by varying the vertical and horizontal distances between UAV and BS

would result in the same amount of DoA estimation errors when UAV are at the altitudes of $10 \mathrm{~m}$ and $20 \mathrm{~m}$, and BS is at the height of $15 \mathrm{~m}$. This phenomenon can be explained as follows: assume UAVs are at the coordinates of $\left[x, y, z_{1}\right]$ and $\left[x, y, z_{2}\right]$ and BS is at the coordinate of $\left[0,0, z_{b}\right]$. From Eq. (15), we can observe that if $\left|z_{1}-z_{b}\right|=\left|z_{2}-z_{b}\right|$, the position error, $[\Delta x, \Delta y, \Delta z]$, would result in the same DoA estimation error, $\Delta \theta$, at points $\left[x, y, z_{1}\right]$ and $\left[x, y, z_{2}\right]$. Thus, when UAVs are at the altitudes of $10 \mathrm{~m}\left(z_{1}\right)$ and $20 \mathrm{~m}\left(z_{2}\right)$, and BS is at the height of $15 \mathrm{~m}\left(z_{b}\right)$, the position of UAVs would satisfy the condition of $\left|z_{1}-z_{b}\right|=\left|z_{2}-z_{b}\right|$, which would lead to the same DoA estimation errors as shown in Fig. 2.

Furthermore, we need to analyze the relationship between DoA error and UAV movement. Towards this aim, we need to firstly exploit Eq. (16) to transfer the velocity and acceleration into the position information and then use Eq. (15) to calculate the DoA. The typical velocity and acceleration errors are around $0.1 \mathrm{~m} / \mathrm{s}$ and $2 \mathrm{~m} / \mathrm{s}^{2}$ [27]. It should be noticed that as the resource allocation slot of cellular-enabled UAV communication is at the millimetre-second level, the velocity error of $0.1 \mathrm{~m} / \mathrm{s}$ and acceleration errors of $2 \mathrm{~m} / \mathrm{s}^{2}$ would have very limited impact on the DoA estimation error. For instance, when the resource allocation slot is $0.05 \mathrm{~s}$, and UAV is flying at the velocity of $44 \mathrm{~m} / \mathrm{s}(160 \mathrm{~km} / \mathrm{h})$ and the acceleration of $10 \mathrm{~m} / \mathrm{s}^{2}$, and with the coordinate of $[50 \mathrm{~m}, 50 \mathrm{~m}, 50 \mathrm{~m}]$, the position error and DoA estimation error caused by $0.1 \mathrm{~m} / \mathrm{s}$ velocity error and $2 \mathrm{~m} / \mathrm{s}^{2}$ acceleration error, are $0.01 \mathrm{~m}$ and $0.0025^{\circ}$, respectively. Compared with the inherent GPS error (up to 20m) and serious DoA estimation error (up to $5^{\circ}$ from Fig. 2), the impact of the inherent mobility error on the performance of the DoA estimation is insignificant.

\section{Navigation Information Correction}

In order to fight against the DoA error caused by UAV mobility, we proposed a hybrid DoA estimation method to exploit the complementary position and channel condition information. Compared with the working mechanism of GUE, navigation and sensor information of UAV devices are required to be transmitted to the ground control centre for decision making, e.g. flight control, forbidden-zone setting, etc. Therefore, different from the traditional beamforming algorithms used in terrestrial communication systems, the position and mobility information are ubiquitously available and could be exploited 
to improve the accuracy of DoA estimation. Instead of solely utilizing GPS position information to obtain DoA angle, we jointly consider the GPS information and channel condition information to design robust beamforming.

For UAV systems, GPS signals are sent by satellites and received by UAVs to estimate its positions. Although GPS satellites use atomic clocks, which are very accurate, the atomic clocks can drift up to a millisecond and results in 10meter level position error in the receiving UAVs. Comparing with GUE, AUEs require more accurate GPS position information for safe operations. Currently, most the commercial UAVs have implemented various algorithms, e.g. Kalman Filter [28], which could reduce 10-meter GPS error to around 2 meters. Depending on the practical implementation, different GPS receivers have different accuracies for position estimation. In order to make the proposed algorithm working for most of the existing GPS devices, 10-meter level GPS error is considered in this study during the algorithm derivation and performance validation.

When UAVs are flying far from the BS, the channel condition-based methods hardly provide accurate DoA estimation. In this situation, GPS and sensor information could be used to calculate the DoA angle. From Fig. 2, relatively small DoA error exists in the position-based DoA estimation in this case. While, when UAVs are flying near to the BS, the inherent position error of GPS system would cause large scale of DoA estimation error. In this situation, directly utilizing GPS information could not obtain the satisfying accuracy of DoA estimation. When UAVs are flying near to the BS, we exploit MUltiple SIgnal Classification (MUSIC) to estimate DoA information. MUSIC algorithm has been widely deployed in the wireless communication system, which calculates the autocorrelation matrix of the received signal and searches the frequency content of the signal autocorrelation matrix in the eigenspace. The stronger signal strength is, the larger frequent content can be searched and the higher accuracy DoA estimation could obtain. In this study, we jointly exploit the MUSIC-driven and position-based approaches to accurately estimate DoA.

The position data in Eqs. (15) and (16) is updated by measuring signal transmission time from the satellite to UAV. The original GPS coordinate will be mapped into the curved surface formed by DoA, as shown in Fig. 3. Let $\left(x_{k}^{e}, y_{k}^{e}, z_{k}^{e}\right)$ and $\left(x_{k}^{c}, y_{k}^{c}, z_{k}^{c}\right)$ denote the original and corrected GPS coordinates, respectively. Through mapping $\left(x_{k}^{e}, y_{k}^{e}, z_{k}^{e}\right)$ to the DoA surface, the corrected position coordinate, $\left(x_{k}^{c}, y_{k}^{c}, z_{k}^{c}\right)$ could be calculated by forming the following optimization problem under the constraint of $\theta \neq 0$,

$$
\begin{aligned}
& \left(x_{k}^{c}, y_{k}^{c}, z_{k}^{c}\right)=\arg \min \sqrt{\left(x_{k}^{c}-x_{k}^{e}\right)^{2}+\left(y_{k}^{c}-y_{k}^{e}\right)^{2}+\left(z_{k}^{c}-z_{k}^{e}\right)^{2}}, \\
& \text { s.t. } \frac{\sqrt{\left(x_{k}^{c}\right)^{2}+\left(z_{k}^{c}-z_{k}^{b}\right)^{2}}}{y_{k}^{c}}=\tan (\theta) .
\end{aligned}
$$

By applying the Lagrange Multiplier [26] to Eq. (17) (see APPENDIX B), the corrected GPS coordinate, $\left(x_{k}^{c}, y_{k}^{c}, z_{k}^{c}\right)$,

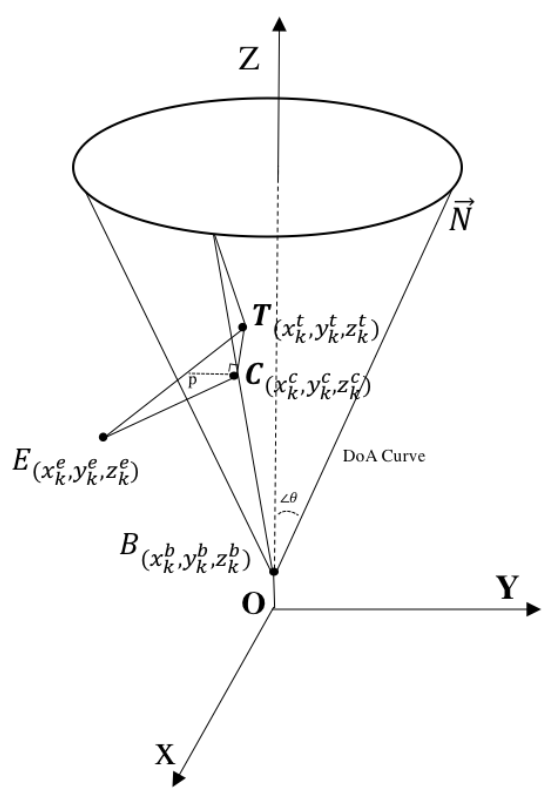

Fig. 3: Navigation information correction in three-dimensional coordinate

can be calculated as

$$
\begin{array}{r}
x_{k}^{c}=\frac{x_{k}^{e} y_{k}^{c}(\tan \theta)^{2}}{y_{k}^{c}\left(1+(\tan \theta)^{2}\right)-y_{k}^{e}}, \\
z_{k}^{c}=\frac{\left(z_{k}^{e}-z_{k}^{b}\right) y_{k}^{c}(\tan (\theta))^{2}}{y_{k}^{c}\left(1+(\tan (\theta))^{2}\right)-y_{k}^{e}}+z_{k}^{b} .
\end{array}
$$

$y_{k}^{c}$ is calculated by the following equation,

$$
y_{k}^{c}=\frac{y_{k}^{e}+\sqrt{\left.\left(\left(x_{k}^{e}\right)^{2}+\left(z_{k}^{e}\right)^{2}\right)(\tan (\theta))^{2}\right)}}{1+(\tan \theta)^{2}} .
$$

The detailed derivation of Eq. (19) is given in the appendix. The optimization problem of Eq. (17) is formulated based on the assumption that $\theta \neq 0$. If $\theta=0$, the corrected coordinate $\left(x_{k}^{c}, y_{k}^{c}, z_{k}^{c}\right)$ could be directly obtained by mapping the original GPS to X-Z plane. In order to analyse the performance of coordinate mapping process, let $\left(x_{k}^{t}, y_{k}^{t}, z_{k}^{t}\right)$ denote the true position of UAV device. Let $\vec{N}$ denote the normal line of the point $T$ that is on the DoA curve. $D_{E T}$ and $E_{C T}$ represent the error distances of the erroneous GPS coordinate and the corrected GPS coordinate. We draw a perpendicular line from the point $C$ of DoA curve, which intersects with the line ET at point $p$. Then the angle formed by line CT and $\mathrm{CP}$ is $90^{\circ}$. The relationship between $E P$ and $C T$ is given by $D_{E T}=D_{E P}+D_{P T}=D_{E P}+D_{C T} / \cos (\varphi)$. As $\cos (\varphi)<90^{\circ}, D_{E T}>D_{C T}$. Denote the angles of $\angle E T C$ and $\angle T E C$ as $\phi$ and $\varphi$. After a series of geometry derivation, the relationship between $E P$ and $C T$ can be quantitatively analyzed from the following equation

$$
\left|\frac{D_{E T}}{D_{C T}}\right|=\frac{\sin (\varphi)+\sin (\phi) \cos (\phi+\varphi)}{\cos (\phi) \cos (\varphi)} .
$$

Eq. (20) demonstrates that the smaller values of $\phi$ and $\varphi$ bring a better effect of coordinate correction. Let $\angle \Theta_{E T N}$ denote the angle of line $\vec{N}$ and line $E T$. The actual values 
of $\phi$ and $\varphi$ is largely determined by $\angle \Theta_{E T N}$. Small value of $\angle \Theta_{E T N}$ results in small values of $\phi$ and $\varphi$, giving better coordinate correction. On the other hand, even if the error GPS coordinate is located in the position far from the normal line $\vec{N}$, the proposed mapping process could still achieve lower DoA error. As both the corrected GPS and true GPS are located on the DoA curve as shown in Fig. 3, we can exploit the UAV movement information (the velocity and the acceleration) to update the corrected GPS to track the UAV, predict the change of DoA angles between two resource allocation slots, and adjust beamforming vector to enhance the signal reception.

\section{PERformance VAlidation AND ANALYSis}

To evaluate the performance of the proposed robust beamforming, we conducted comprehensive simulation experiments. The parameter configuration is summarised in Table I. According to commercial UAV products [29], GPS signals are generated at the frequency of $20 \mathrm{~Hz}$ and with the error $10 \mathrm{~m}$. Without loss of generality, the Rural Macro (RMa) channel scenario defined by 3GPP TR 38.901 [30] is used in this study to generate the $\mathrm{A} 2 \mathrm{G}$ links. Totally up to 300 rays were generated in this simulation, each of which is allocated with the different delays, power and DoA angles. The power distribution is generated by the exponential delay distribution, and power distribution given by [30]. During the channel simulation, 300 rays are grouped into 10 clusters, each of which includes 30 rays. The first cluster is to simulate the LoS path and the remaining nine clusters are used to model the NLoS path. To obtain a deep understanding of the features of UAV A2G channel, the power distribution of LoS and NLoS is added in the revised manuscript, as shown in Fig. 4. From Fig. 4, it can be seen that the majority of transmission power (around 93\%) is located in the first cluster, revealing that LoS dominates the overall channel response in UAV scenario. In addition, there are three UAVs modelled in the simulation, the interesting UAV (UAV1), and two interference UAVs (UAV2 and UAV3). To capture the characteristics of the real-world cellular-enabled UAV communication, UAV1 is allocated in the serving BS and UAV2 and UAV3 are assigned in the neighbouring BSs to generate the interference signals. The Semi-Random Circular Movement (SRCM) mobility [31] is adopted in the simulation to update the UAV positions at each transmission slot. SRCM is an important UAV mobility model, mainly used in the scenarios where a potential target location is known, and UAVs are dispatched to collect information in the nearby area, e.g. search and rescue. The results are collected and averaged from 100 runs, each of which lasts 120 resource allocation slot. Because the GPS measurement work [32] [33] adapts $20 \mathrm{~Hz}$ as the frequency sampling, each allocation slot is set to be 50 millimetre-seconds (ms) in the simulation experiments. Six benchmark algorithms are chosen to conduct performance comparison, which include optimal beamforming algorithm, Linearly Constrained Minimum Variance (LCMV) algorithm, Position-based beamforming algorithm, Diagonal Loading (DL) algorithm, Robust Capon Beamforming (RCB) and Robust LCMV algorithm. The aim of adapting the optimal
TABLE I: Simulation parameter configuration

\begin{tabular}{ll}
\hline \hline Parameters & \multicolumn{1}{c}{ Values } \\
\hline Carrier frequency $\left(f_{c}\right)$ & $2.4 \mathrm{GHz}$ \\
Light speed $(c)$ & $3.0 * 10^{8} \mathrm{~m} / \mathrm{s}$ \\
Signal wavelength $(\lambda)$ & $c / f_{c}$ \\
Number of Antennas at BS $(N)$ & $8,16,32,64$ \\
UAV speed $(V)$ & $160 \mathrm{~km} / \mathrm{h}$ \\
Flight heights $\left(h_{A U E(1,2,3)}\right)$ & $50 \mathrm{~m}, 100 \mathrm{~m}, . ., 250 \mathrm{~m}, 300 \mathrm{~m}$ \\
Flight heights $\left(h_{B S}\right)$ & $15 \mathrm{~m}$ \\
Number of interfering UAVs $(N)$ & 2 \\
UAV transmission power $\left(P_{s}\right)$ & $23 \mathrm{dBm}$ \\
Antenna inter-element distance $(d)$ & $\lambda / 2$ \\
Signal-to-Noise-Ratio $(S N R)$ & $10 \mathrm{~dB}$ \\
Signal-to-Interference-Ratio $(S I R)$ & $2 \mathrm{~dB}$ \\
MUSIC Search Resolution $(S e a r c h)$ & $0.1^{\circ}$ \\
GPS Error $\left(G P S_{e}\right)$ & $10 \mathrm{~m}$ \\
Symbol Length $\left(T_{B}\right)$ & $66.7 * 10^{-6} \mathrm{~s}$ \\
Sampling Interval $\left(T_{S}\right)$ & $0.03255 * 10^{-9} \mathrm{~s}$ \\
Bandwidth $(B)$ & $5 \mathrm{MHz}$ \\
Channel Model $(h)$ & $\mathrm{RMa} \mathrm{Scenario} \mathrm{defined} \mathrm{in}[30]$ \\
\hline
\end{tabular}

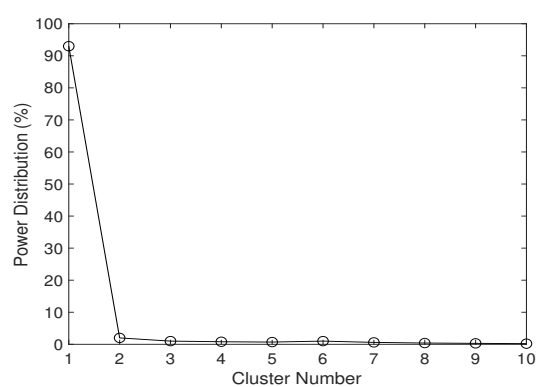

Fig. 4: Power distribution of 3GPP A2G channel model

beamforming algorithm in the simulation experiments is to obtain the upper-bound beamforming performance, which is meaningful for conducting the performance comparison and obtaining a comprehensive understanding of the beamforming design. The performance of different algorithms is analysed in terms of DoA error, interference power, noise power and output SINR,

In Fig. 5, we investigate the DoA error, $\Delta \theta$, of UAV1 for different beamforming algorithms. $\Delta \theta$ is defined as the absolute difference between real DoA and estimated DoA, $\Delta \theta=\left|\theta^{t}-\theta^{e}\right|$. We can see that the proposed algorithm with the position correction approach shows a significant gain over the well-known LCMV approach in [34] and the position-based beamforming algorithm, the inherent position error of which is not corrected. Compared with the other two approaches, the proposed method could achieve a reduction of 90-99\% DoA error. In addition, all three algorithms show the periodic changes. This is because DoA error is largely determined by the UAV flight status. As introduced in the previous paragraph, the flight path is generated by the SRCM model and UAV1 are flying around a certain point. After a certain amount of flight time, UAV1 would finish one round search and start the next round. DoA is closely related to the direction of the signal sent out and the DoA error has a similar change pattern as the mobility model.

In Figs. 6 and 7, we investigate the performance of the 


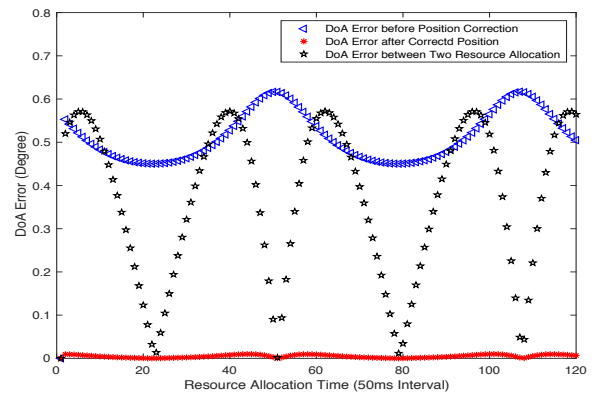

Fig. 5: DoA error of the interesting UAV1

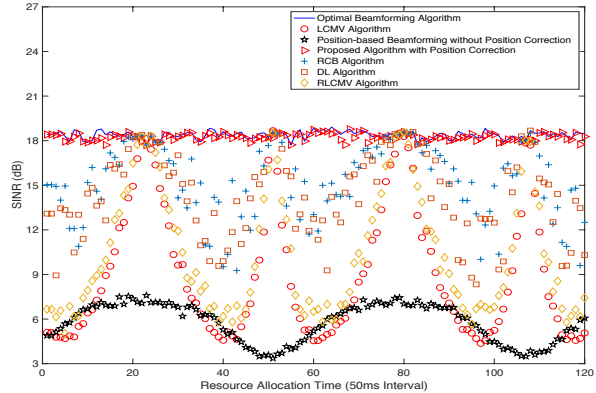

Fig. 6: Ouput SINR comparisons of different beamforming algorithms

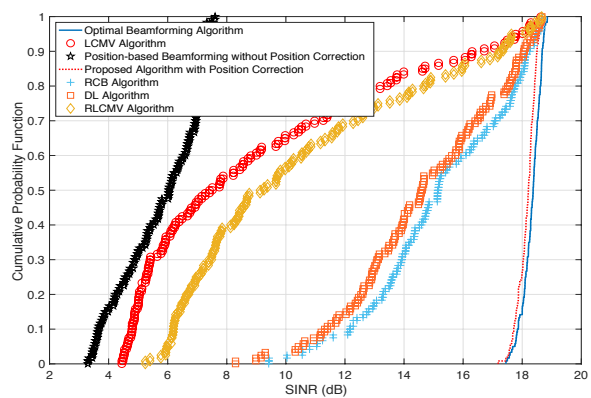

Fig. 7: Cumulative probability function of the output SINR of different beamforming algorithms

achieved SINR of the proposed algorithm. In order to clearly show the performance gains, the real DoA values are used in the optimal beamforming algorithm to calculate the optimal beamforming performance. Fig. 6 shows that the proposed algorithm outperforms the other five approaches. Due to the significant reduction of DoA error, the proposed robust beamforming has least-error position information for calculating the steering vector in Eq. (2) and beamforming weight vector in Eq. (9), thus achieving good SINR output. In addition, as shown in Fig. 6, LCMV, RCB, DL and robust LCMV can occasionally achieve good SINR performance at the resource allocation time of 30th, 60th, 90th and other time slots (the interval of 30 slots), but could not provide stable SINR output. The reason for this phenomenon is that when UAV is operated in the vertical direction of BS, slight DoA error is introduced between two resource allocation slots and good performance of the receiving SINR could be achieved, however, when UAV changes its flight status according to the mission requirements, the large scale of DoA error would be generated, which results in low receiving SINR. In this case, the position correction process proposed in this work can be used to reduce the DoA error and achieve good and stable receiving SINR.

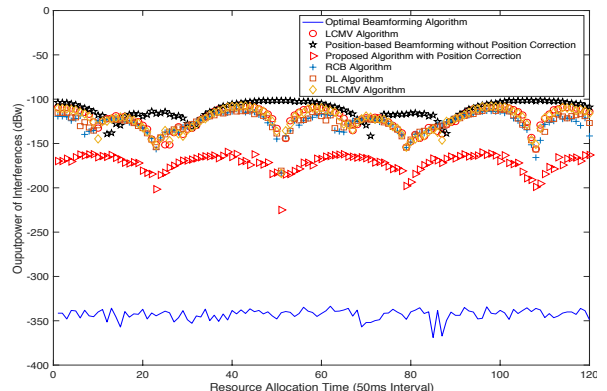

Fig. 8: Comparison of the output interference power for different beamforming algorithms

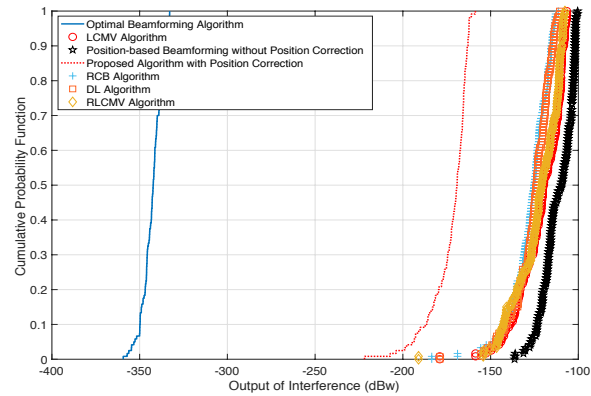

Fig. 9: Cumulative probability function of the output interference power for different beamforming algorithms

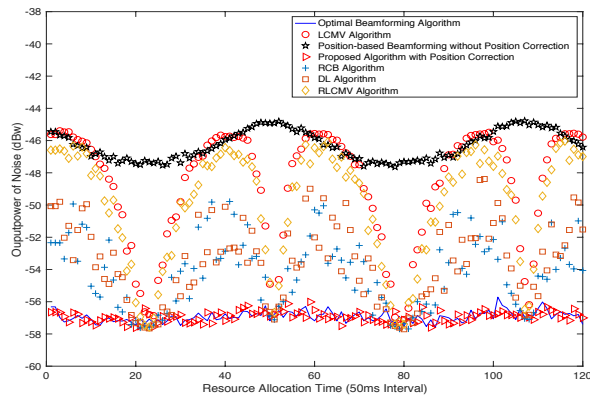

Fig. 10: Comparison of the output noise power for different beamforming algorithms

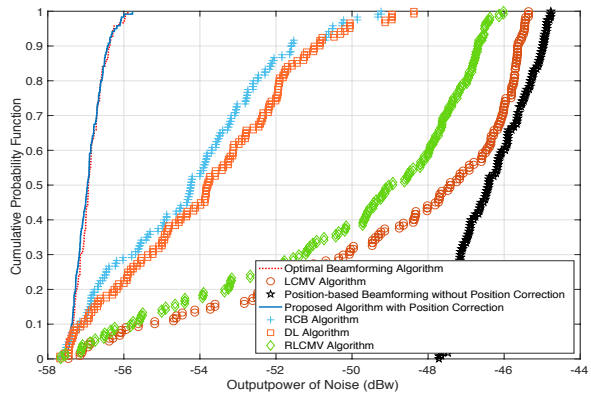

Fig. 11: Cumulative probability function of the output noise power for different beamforming algorithms

In Figs. 8 and 9, we investigate the performance of the proposed algorithm in terms of interference cancellation. The results show that the proposed algorithm has lower interference responses compared with the other five algorithms. As shown in Eq. (8), the first constraint condition guarantees that the signal from interesting UAVs could be responded without any power loss after multiplying the beamforming weight solution $\omega$. And the second optimization constraint guarantees that the interfering signals are blocked by the beamformer through setting the null points in the direction of interfering sources. In 
TABLE II: Output SINR of the proposed algorithm (dB)

\begin{tabular}{ccccccc}
\hline & $50 \mathrm{~m}$ & $100 \mathrm{~m}$ & $150 \mathrm{~m}$ & $200 \mathrm{~m}$ & $250 \mathrm{~m}$ & $300 \mathrm{~m}$ \\
\hline $\mathrm{N}=8$ & -3.6113 & 10.7829 & 14.2031 & 15.1893 & 15.4845 & 15.5643 \\
$\mathrm{~N}=16$ & 10.7983 & 17.9725 & 18.0841 & 18.1704 & 18.1896 & 18.1925 \\
$\mathrm{~N}=32$ & 17.8571 & 18.8746 & 19.2446 & 19.3299 & 19.3403 & 19.3734 \\
$\mathrm{~N}=64$ & 18.9302 & 19.4574 & 19.7396 & 19.7921 & 19.8049 & 19.8145 \\
\hline
\end{tabular}

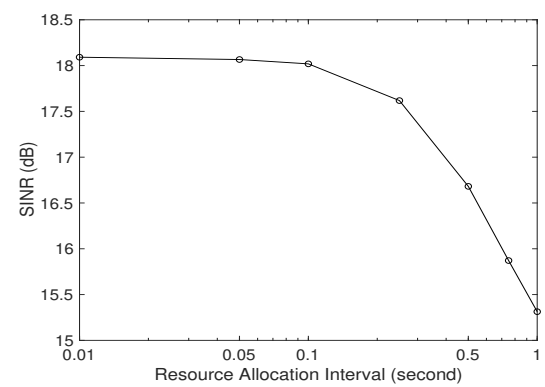

Fig. 12: The impact of the interval length on the performance of the proposed algorithm

addition, the optimal beamforming algorithm achieves the best performance in terms of interference cancellation. Because DoA error would result in the leakage of interfering power and the optimal beamforming algorithm does not suffer from any DoA error, and therefore it achieves the best performance for interference cancellation.

Furthermore, the performance of noise minimization is also investigated in Figs. 10 and 11. The output SINR is not only related to the output signal and interference power, but also determined by the level of output noise power, as described in Eq. (5). As shown in Fig. 10, the proposed algorithm has lower noise power (around 10dBm) compared with the LCMV and the position-based beamforming algorithms. Instead of minimizing the output power adopted by LCMV algorithm, the proposed algorithm split the minimization of the output power into three aspects, cancelling the interference, guaranteeing the interesting signal and minimizing the output noise power as shown in Eq. (8). As the receiving noise is independent with the receiving signal, then BS could utilize the Noise Analyser or Meter (NFM) to measure the noise figure, calculate the noise autocorrelation matrix, $R_{n}$, and design effective beamforming vector to minimize the output noise $\omega^{H} R_{n} \omega$. It can be seen that the proposed algorithm achieves a similar level of output noise power as that of the optimal beamforming algorithm.

In addition, the antenna model and AUE altitude would have significant impacts on the performance of the proposed robust beamforming. Therefore, we investigate the output SINR performance by varying the antenna numbers $(8,16$, 32 and 64) and AUE altitudes (from $50 \mathrm{~m}$ to $300 \mathrm{~m}$ with an interval of 50m). The simulation results are shown in Table. II. From the simulation results in each column of the table, it can be seen that the increase of the antenna number is benefitable to the output SINR improvement. This is because the mainlobe width of the antenna beamforming is largely determined by the antenna number. The increased number of the antenna enables the BS to narrow the beam width and increase the antenna response in the direction of the signal source, thus improving the output SINR. Furthermore, through comparing the SINR in each row of Table. II, we can observe that the performance of the proposed algorithm is enhanced with the increase of the AUE altitudes from $50 \mathrm{~m}$ to $300 \mathrm{~m}$. This is because the A2G channel consists of both LoS and NLoS. The higher UAV altitude would lead to the more transmission power to be allocated to the LoS paths, which brings the enhanced performance with respect to the output SINR for the proposed algorithm.

The interval length would affect the accuracy of the designed algorithm. In order to investigate the relationship between the accuracy of the proposed solution and the interval length, we conducted experiments through varying the length of the interval from $0.01 \mathrm{~s}$ to $1 \mathrm{~s}$ and the simulation results are drawn in Fig. 12. It can be seen that, with the increase of the interval length from $0.01 \mathrm{~s}$ to $0.1 \mathrm{~s}$, the proposed algorithm can obtain stable performance with respect to the output SINR. While from $0.1 \mathrm{~s}$ to $1 \mathrm{~s}$, there is slight performance degradation for the proposed algorithm. This is because the proposed position-based beamforming exploits velocity and acceleration information to track the movement of UAV, predict the changes of UAV, and adjust the beamforming weights for signal reception enhancement. In this context, the velocity and acceleration information can be obtained at a very high frequency, such as $250 \mathrm{~Hz}$ [35]. If the interval length is long such as $1 \mathrm{~s}$, the velocity and acceleration used in the position prediction may be out-of-date, resulting in the performance degradation for the proposed position-based beamforming design. Therefore, there is a need to ensure the resource allocation slot is small than $100 \mathrm{~ms}$ to implement the proposed algorithm in the practical UAV system.

\section{CONCLUSION}

A novel position-based robust beamforming algorithm was proposed in this study to enhance the performance of the cellular-enable UAV communication. To mitigate the serious interference generated by UAVs in cellular networks, we proposed a new beamforming optimization approach to maximize the system throughput under the constraint of interference and noise minimization, which was solved by exploiting the Lagrange multiplier approaches. The implementation of the optimal beamforming is largely determined by the accuracy of DoA estimation. While the rapid velocity changes of UAV devices make it difficult to achieve accurate position information. To address this issue, we developed a mapping approach to iteratively utilize the MUSIC algorithm to correct the position information and exploit the updated position information to predict the DoA information. By making full use of two complementary information resources, the proposed algorithm is able to enhance the received signal power, suppress interferences and noises, thus improving the receiving SINR. Comprehensive simulation experiments showed that the proposed algorithm outperforms the Linearly Constrained Minimum Variance (LCMV) algorithm, Position-based beamforming algorithm, Diagonal Loading (DL) algorithm, Robust Capon Beamforming (RCB) and Robust LCMV algorithm. In 
addition, the proposed algorithm can be deployed in the current and future cellular system to enhance the communication quality of UAVs and mitigate their strong interference to other ground and aerial users.

\section{ACKNOWLEDGEMENTS}

This work was partly supported by the EU Horizon 2020 INITIATE project under the Grant Agreement No. 101008297.

\section{APPENDIX A \\ DETERMINATION OF EQ. (9).}

In order to find the solve the optimization problem in Eq. (8), we form the Lagrangian function as follows,

$$
\mathrm{Ł}\left(\omega^{H}, \lambda\right)=\omega R_{n} \omega+\lambda\left(\omega^{H} A(\theta)-F\right),
$$

where $F=[1,0, \ldots, 0]$. Then,

$$
\frac{\partial \succeq\left(\omega^{H}, \lambda\right)}{\partial \omega^{H}}=R_{n} \omega+\lambda A(\theta)=0,
$$

and

$$
\frac{\partial \succeq\left(\omega^{H}, \lambda\right)}{\partial \lambda}=\omega^{H} A(\theta)-F=0 .
$$

Through jointly solving Eqs. (22) and (23), the beamforming weight vector, $\omega$, could be calculated by

$$
\omega=-\left[A(\theta)^{H} R_{n}{ }^{-1} A(\theta)\right]^{-1} R^{-1} A(\theta) F .
$$

\section{APPENDIX B}

DETERMINATION OF EQ. (18) AND EQ. (19).

To solve the optimization problem of Eq. (17), instead of directly working out the optimization problem of this equation, we transfer the Eq. (17) to the following optimization problem,

$\left(x_{k}^{c}, y_{k}^{c}, z_{k}^{c}\right)=\arg \min \left(x_{k}^{c}-x_{k}^{e}\right)^{2}+\left(y_{k}^{c}-y_{k}^{e}\right)^{2}+\left(z_{k}^{c}-z_{k}^{e}\right)^{2}$ s.t. $\left(x_{k}^{c}\right)^{2}+\left(z_{k}^{c}-z_{k}^{b}\right)^{2}=\left(y_{k}^{c}\right)^{2}(\tan (\theta))^{2}$.

Applying the Lagrange multiplier to Eq. (25) and defining the Lagrangian expression as follows,

$$
\begin{aligned}
& \mathrm{\iota}\left(x_{k}^{c}, y_{k}^{c}, z_{k}^{c}, \eta\right)=\left(x_{k}^{c}-x_{k}^{e}\right)^{2}+\left(y_{k}^{c}-y_{k}^{e}\right)^{2}+ \\
& \left(z_{k}^{c}-z_{k}^{e}\right)^{2}+\eta\left(\left(x_{k}^{c}\right)^{2}+\left(z_{k}^{c}-z_{z k}^{b}\right)^{2}-\left(y_{k}^{c}\right)^{2}(\tan \theta)^{2}\right),
\end{aligned}
$$

where $\eta$ is a Lagrange multiplier. Exploiting the feature of Lagrange multiplier and solving $\nabla_{x_{k}^{c}, y_{k}^{c}, z_{k}^{c}, \eta} \mathbf{L}\left(x_{k}^{c}, y_{k}^{c}, z_{k}^{c}, \eta\right)=$ 0 can achieve the following three equations,

$$
\begin{array}{r}
\frac{\partial \mathrm{Ł}\left(x_{k}^{c}, y_{k}^{c}, z_{k}^{c}, \eta\right)}{\partial x_{k}^{c}}=x_{k}^{c}-x_{k}^{e}+\eta x_{k}^{c}=0 \\
=>\eta=\frac{x_{k}^{e}}{x_{k}^{c}}-1, \\
\frac{\partial \succeq\left(x_{k}^{c}, y_{k}^{c}, z_{k}^{c}, \eta\right)}{\partial y_{k}^{c}}=y_{k}^{c}-y_{k}^{e}-\eta y_{k}^{c}(\tan (\theta))^{2}=0 \\
=>\eta=\frac{y_{k}^{c}-y_{k}^{e}}{y_{k}^{c}(\tan (\theta))^{2}},
\end{array}
$$

and

$$
\begin{array}{r}
\frac{\partial \mathrm{\Perp}\left(x_{k}^{c}, y_{k}^{c}, z_{k}^{c}, \eta\right)}{\partial z_{k}^{c}}=z_{k}^{c}-z_{k}^{e}+\eta\left(z_{z}^{c}-z_{z}^{b}\right)=0 \\
=>\eta=\frac{z_{k}^{e}-z_{k}^{c}}{z_{k}^{c}-z_{k}^{b}} .
\end{array}
$$

By linking Eqs. (27) and (28) through $\eta, x_{k}^{c}$ could be expressed as a function of $y_{k}^{c}$,

$$
\begin{aligned}
& \frac{x_{k}^{e}}{x_{k}^{c}}=\frac{y_{k}^{c}-y_{k}^{e}+y_{k}^{c}(\tan (\theta))^{2}}{y_{k}^{c}(\tan (\theta))^{2}} \\
& =>x_{k}^{c}=\frac{x_{k}^{e} y_{k}^{c}(\tan (\theta))^{2}}{y_{k}^{c}\left(1+(\tan (\theta))^{2}\right)-y_{k}^{e}} .
\end{aligned}
$$

Similar to the derivation of $x_{k}^{c}, z_{k}^{c}$ could also be denoted as the function of $y_{k}^{c}$ as follows:

$$
z_{k}^{c}=\frac{\left(z_{k}^{e}-z_{k}^{b}\right) y_{k}^{c}(\tan (\theta))^{2}}{y_{k}^{c}\left(1+(\tan (\theta))^{2}\right)-y_{k}^{e}}+z_{k}^{b} .
$$

By taking $x_{k}^{c}, y_{k}^{c}$ and $z_{k}^{c}$ into the optimization constraint condition of Eq. (25), $\left(x_{k}^{c}\right)^{2}+\left(z_{z}^{c}\right)^{2}=\left(y_{k}^{c}\right)^{2}(\tan (\theta))^{2}, y_{k}^{c}$ could be calculated in the following equation,

$\left[\frac{x_{k}^{e} y_{k}^{c}(\tan (\theta))^{2}}{y_{k}^{c}\left(1+(\tan (\theta))^{2}\right)-y_{k}^{e}}\right]^{2}+\left[\frac{\left(z_{k}^{e}-z_{k}^{b}\right) y_{k}^{c}(\tan (\theta))^{2}}{y_{k}^{c}\left(1+(\tan (\theta))^{2}\right)-y_{k}^{e}}+z_{k}^{b}\right]^{2}$

After a series of derivation, $y_{k}^{c}$ could be calculated as $y_{k}^{c}=$ $\frac{-B \pm \sqrt{B^{2}-4 A C}}{2 A}$ under the constraint of $B^{2}-4 A C \geq 0$, where $A, B$, and $C$ are calculated by,

$$
\begin{gathered}
A=\left(1+(\tan (\theta))^{2}\right), \\
B=-2 y_{k}^{e}\left(1+(\tan (\theta))^{2}\right),
\end{gathered}
$$

and

$$
C=\left(y_{k}^{e}\right)^{2}-\left(\left(x_{k}^{e}\right)^{2}+\left(z_{k}^{e}\right)^{2}\right)(\tan (\theta))^{2} .
$$

\section{REFERENCES}

[1] E. Ackerman and E. Strickland. Medical delivery drones take flight in east africa. IEEE Spectrum, 55(1):34-35, Jan. 2018

[2] S. Minaeian, J. Liu, and Y. Son. Vision-based target detection and localization via a team of cooperative uav and ugvs. IEEE Transactions on Systems, Man, and Cybernetics: Systems, 46(7):1005-1016, Jul. 2016.

[3] W. Miao, C. Luo, G. Min, and Z. Zhao. Lightweight 3-d beamforming design in $5 \mathrm{~g}$ uav broadcasting communications. IEEE Transactions on Broadcasting, 66(2):515-524, May. 2020.

[4] W. Mei and R. Zhang. Cooperative downlink interference transmission and cancellation for cellular-connected uav: A divide-and-conquer approach. IEEE Transactions on Communications, 68(2):1297-1311, Feb. 2020.

[5] R. Amer, W. Saad, and N. Mar.etti. Toward a connected sky: Performance of beamforming with down-tilted antennas for ground and uav user co-existence. IEEE Communications Letters, 23(10):1840-1844, Oct. 2019

[6] R. Amer, W. Saad, and N. Mar.etti. Mobility in the sky: Performance and mobility analysis for cellular-connected uavs. IEEE Transactions on Communications, 68(5):3229-3246, May. 2020.

[7] C. D'Andrea, A. Garcia-Rodriguez, G. Geraci, L. G. Giordano, and S. Buzzi. Analysis of uav communications in cell-free massive mimo systems. IEEE Open Journal of the Communications Society, 1:133147, Jan. 2020 
[8] R. Amer, W. Saad, B. Galkin, and N. Mar.etti. Performance analysis of mobile cellular-connected drones under practical antenna configurations. arxiv, 2020

[9] X. Gao, J. Zhang, J. Jin, and Z. Wang. Optimal diagonal power loading minimizing asymptotic symbol error probability for distributed mimo systems with zf receivers. IEEE Transactions on Vehicular Technology, 65(9):7818-7823, Sep. 2016

[10] S. D. Somasundaram. Linearly constrained robust capon beamforming. IEEE Transactions on Signal Processing, 60(11):5845-5856, Nov. 2012

[11] J. Xu, G. Liao, S. Zhu, and L. Huang. Response vector constrained robust lcmv beamforming based on semidefinite programming. IEEE Transactions on Signal Processing, 63(21):5720-5732, Nov. 2015.

[12] W. Miao, G. Min, Y. Wu, H. Wang, and J. Hu. Performance modelling and analysis of software-defined networking under bursty multimedia traffic. ACM Trans. Multimedia Comput. Commun. Appl., 12(5s), Sep. 2016.

[13] A. Bonfante, L. Galati Giordano, D. López-Pérez, A. Garcia-Rodriguez, G. Geraci, P. Baracca, M. M. Butt, and N. Marchetti. $5 \mathrm{~g}$ massive mimo architectures: Self-backhauled small cells versus direct access. IEEE Transactions on Vehicular Technology, 68(10):10003-10017, Oct. 2019.

[14] W. Miao, G. Min, Y. Wu, H. Huang, Z. Zhao, H. Wang, and C. Luo Stochastic performance analysis of network function virtualization in future internet. IEEE Journal on Selected Areas in Communications, 37(3):613-626, Jan. 2019.

[15] H. Huang, W. Miao, G. Min, C. Huang, X. Zhang, and C. Wang. Resilient range-based d-dimensional localization for mobile sensor networks. IEEE/ACM Transactions on Networking, pages 1-14, Jul. 2020.

[16] K. Xiong, B. Wang, C. Jiang, and K. J. R. Liu. A broad beamforming approach for high-mobility communications. IEEE Transactions on Vehicular Technology, 66(11):10546-10550, Nov. 2017.

[17] X. Li, Y. Zhu, and P. Xia. Enhanced analog beamforming for single carrier millimeter wave mimo systems. IEEE Transactions on Wireless Communications, 16(7):4261-4274, Jul. 2017.

[18] N. K. D. Venkategowda, N. Tandon, and A. K. Jagannatham Mvdr-based multicell cooperative beamforming techniques for unicast/multicast mimo networks with perfect/imperfect csi. IEEE Transactions on Vehicular Technology, 64(11):5160-5176, Nov. 2015.

[19] A. Koubaa and B. Qureshi. Dronetrack: Cloud-based real-time object tracking using unmanned aerial vehicles over the internet. IEEE Access, 6:13810-13824, Mar. 2018

[20] X. Chen, J. Lu, S. Liu, and P. Fan. Location-aided umbrella-shaped massive mimo beamforming scheme with transmit diversity for high speed railway communications. In 2016 IEEE 83rd Vehicular Technology Conference (VTC Spring), May. 2016.

[21] Qualcomm Technologies. Inc. Lte unmanned aircraft systems. Trial Report, v1.0.1, May. 2017.

[22] X. Lin, V. Yajnanarayana, S. Muruganathan, S. Gao, H. Asplund, H. Maattanen, A. MattiasBergström, Sebastian Euler, and Y.-P. Eric Wang. The sky is not the limit: Lte for unmanned aerial vehicles. IEEE Communications Magazine, 56(4):204-210, Apr. 2018.

[23] W. Khawaja, I. Guvenc, D. W. Matolak, U. Fiebig, and N. Schneckenburger. A survey of air-to-ground propagation channel modeling for unmanned aerial vehicles. IEEE Communications Surveys Tutorials, 21(3):2361-2391, Third Quarter 2019.

[24] M. Zhang and Y. Liu. Secure beamforming for untrusted miso cognitive radio networks. IEEE Transactions on Wireless Communications, 17(7):4861-4872, Jul. 2018.

[25] C. E. Shannon. A mathematical theory of communication. The Bell System Technical Journal, 27(3):379-423, Jul. 1948.

[26] L. Griva, S. G. Nash, and A. Sofer. Linear and Nonlinear Programming. Second Edition, Cambridge University Press, 2009.

[27] E. Zhang and N. Masoud. Increasing gps localization accuracy with reinforcement learning. IEEE Transactions on Intelligent Transportation Systems, Early Acces.

[28] S. Miura, L. Hsu, F. Chen, and S. Kamijo. Gps error correction with pseudorange evaluation using three-dimensional maps. IEEE Transactions on Intelligent Transportation Systems, 16(6):3104-3115, Dec. 2015.

[29] SatLab Geosolutions. Uav/rtk automated gnss sensor. [Online] http://www.satlabgps.com/en/products/uavuas/uavrtk-automated-gnsssensoraspx.

[30] 3GPP TR 38.901. Study on channel model for frequencies from 0.5 to 100 ghz. [Online] ftp://www.3gpp.org/specs/archive/38 series/38.901, May. 2017.

[31] J. Xie, Y. Wan, J. H. Kim, S. Fu, and K. Namuduri. A survey and analysis of mobility models for airborne networks. IEEE Communications Surveys Tutorials, 16(3):1221-1238, Third Quarter 2014.
[32] C. Specht. Diagnostics of the tram track shape with the use of the global positioning satellite systems (gps/glonass) measurements with a $20 \mathrm{hz}$ frequency sampling. Journal of Vibroengineering, 16(6):30763085, Sep. 2014.

[33] J. Hwang, H. Yun, Y. Suh, J. Cho, and D. Lee. Development of an rtkgps positioning application with an improved position error model for smartphones. Journal of Vibroengineering, 12(10):12988-13001, Sep. 2012.

[34] S. Chakrabarty and E. A. P. Habets. On the numerical instability of an lcmv beamformer for a uniform linear array. IEEE Signal Processing Letters, 23(2):272-276, Feb. 2016.

[35] M. Potter, L. Ojeda, N.Perkins, and S.Cain. Effect of imu design on imu-derived stride metrics for running. Sensor, 19(11):2601, Jun. 2019.

Wang Miao received his Ph.D. degree in Computer Science from the University of Exeter, United Kingdom in 2017. He is currently a Postdoctoral Research Associate at the College of Engineering, Mathematics, and Physical Sciences of the University of Exeter. His research interests focus on Vehicle Edge Computing, Artificial Intelligence, Network Function Virtualization, Software Defined Networking, Unmanned Aerial Networks, Wireless Communication Networks, and Performance Modelling and Analysis.

Chunbo Luo received the Ph.D. degree in high-performance cooperative wireless networks from the University of Reading, Reading, U.K. His research has been supported by NSFC, Royal Society, EU H2020, and industries. His research interest focuses on developing model-based and machine learning algorithms to solve networking and engineering problems, such as wireless networks, with a particular focus on unmanned aerial vehicles. Dr. Luo is a Fellow of the Higher Education Academy.

Geyong Min is a Professor of High Performance Computing and Networking in the Department of Computer Science within the College of Engineering, Mathematics and Physical Sciences at the University of Exeter, United Kingdom. He received the $\mathrm{PhD}$ degree in Computing Science from the University of Glasgow, United Kingdom, in 2003, and the B.Sc. degree in Computer Science from Huazhong University of Science and Technology, China, in 1995. His research interests include Future Internet, Computer Networks, Wireless Communications, Multimedia Systems, Information Security, High Performance Computing, Ubiquitous Computing, Modelling and Performance Engineering.

Yang Mi received the B.Eng. degrees from Beijing Institute of Technology, China, in 2016. She is currently a PhD student at the College of Engineering, Mathematics, and Physical Sciences of the University of Exeter, UK. Her research interests include visual retrieval, video coding, and saliency attention.

Zhengxin Yu received the M.Sc. degree in information technology management for business from the University of Exeter, U.K., in 2016, where she is currently pursuing the Ph.D. degree with the Department of Computer Science, College of Engineering, Maths, and Physical Science. Her research interests include deep learning, federated learning, and mobile edge computing. 